\title{
EFEITOS DA INTERAÇÃO HUMANO-ANIMAL NO BEM-ESTAR DE RUMINANTES LEITEIROS: UMA REVISÃO
}

\author{
Mayara Andrioli ${ }^{1}$ \\ Monique Carvalhal ${ }^{2}$ \\ Franciely Costa $^{2}$ \\ Mateus José Rodrigues Paranhos da Costa ${ }^{3}$
}

\begin{abstract}
RESUMO
O bem-estar dos animais de produção vem recebendo crescente atenção à medida que os consumidores têm se mostrado mais preocupados com a maneira como os animais de produção são tratados. Nesse sentido, a interação humano-animal tem importância na produção agropecuária, pois a preocupação com o bem-estar animal está diretamente relacionada à melhoria na rotina de manejo das fazendas. Além disso, é comprovado cientificamente que a relação humano-animal tem potencial para causar impactos positivos ou negativos na produtividade e no bem-estar das diferentes espécies dos animais de produção. Dessa maneira, a relação entre a interação humano-animal e o bem-estar dos animais de produção leiteira são discutidos nessa revisão, com o propósito de mostrar a importância da implantação das boas práticas no manejo de ruminantes leiteiros.
\end{abstract}

Palavras-chave: boas práticas de manejo, comportamento, produção de leite.

\section{EFFECTS OF HUMAN-ANIMAL INTERACTIONS ON THE WELFARE OF DAIRY RUMINANTS: A REVIEW}

\begin{abstract}
The welfare of farm animals has been receiving increasingly attention from consumers, as they have been more concerned about the way that farm animals are treated. In this sense, human-animal interaction has an important role in livestock production, since the concern about farm animal welfare is directly related to the improvement in the farm management routines. Additionally, it is scientifically proven that the human-animal relationship has the potential to cause positive or negative impacts on the productivity and welfare of farm animals. The relationship between human-animal interaction and the welfare of dairy ruminants is addressed in this review, with the purpose of showing the importance of implementing good practices of handling of dairy ruminants.
\end{abstract}

Key-words: good practices of handling, behavior, milk production.

\footnotetext{
${ }^{1}$ Faculdade de Ciências Agrárias e Veterinárias FCAV - UNESP. Correspondência: may.andrioli6@ gmail.com

2 Grupo de Estudos em Etologia e Ecologia Animal - ETCO/UNESP FCAV. monique_carvalhal@ hotmail.com

${ }^{3}$ Professor Adjunto no Departamento de Zootecnia da Faculdade de Ciências Agrárias e Veterinárias - UNESP, Grupo de Estudos em Etologia e Ecologia Animal - ETCO/UNESP FCAV. mateus.paranhos@ unesp.br
} 


\section{EFECTOS DE LA INTERACCIÓN HUMANO-ANIMAL EN EL BIENESTAR DE RUMIANTES LECHEROS: UNA REVISIÓN}

\section{RESUMEN}

El bienestar de los animales recibe cada vez más atención por los consumidores, que están preocupados por cómo los animales de producción son tratados. En este sentido, la interacción humano-animal es importante en la producción ganadera, ya que la preocupación con el bienestar animal está directamente relacionada con la mejora en la rutina de manejo de las granjas. Además, está científicamente comprobado que la relación humano-animal tiene un impacto en la productividad y el bienestar de las diferentes especies de animales de producción. Por lo tanto, tratamos de discutir la relación entre la interacción humano-animal y el bienestar de los animales de producción de leche en esta revisión, con el objetivo de mostrar la importancia de la implementación de buenas prácticas de manejo en los sistemas de producción ganadera.

Palabras-clave: buenas prácticas de manejo, comportamiento, producción de leche.

\section{INTRODUÇÃO}

Atualmente, as cadeias produtivas da pecuária enfrentam desafios relacionados a uma série de demandas dos consumidores, dentre elas a crescente demanda por produtos de origem animal que sejam resultantes de sistemas de produção sustentáveis e que assegurem boas condições de bem-estar aos animais (1). Para enfrentar esses desafios é necessário adequar as técnicas de criação aos princípios de sustentabilidade (econômico, social e ambiental) e adotar princípios éticos de respeito aos animais de produção e estratégias que assegurem que seu bem-estar é bom $(2,3)$. O desafio é desenvolver e implementar estratégias que proporcionem boas condições de bem-estar aos animais em todas as fases de criação, sem causar danos ao ambiente e que assegurem rentabilidade aos produtores.

Nas cadeias produtivas do leite, envolvendo principalmente as espécies bovina, caprina, ovina e bubalina há um desafio maior, o de garantir boas condições de bem-estar aos animais que geralmente são criados em sistemas intensivos de produção e passam muito tempo em estreito contato com humanos, principalmente quando submetidos a ordenha. Esse manejo diário, quando mal realizado, pode resultar em situações que prejudicam o bem-estar dos animais. Entretanto, por outro lado, quando bem feito, pode resultar em maior proximidade entre humanos e animais que permitem, por exemplo, redução do estresse e maior facilidade para a realização dos manejos e para a detecção de problemas de saúde.

Há evidências claras de que a qualidade da interação humano-animal tem importante papel na definição do bem-estar dos animais e na expressão de seus potenciais produtivos (4). Esta constatação tem impulsionado o desenvolvimento e implementação de práticas de manejo que estimulem a adoção de ações positivas durante a realização das rotinas de trabalho, com a expectativa de que essas ações promovam o bem-estar e a produtividade dos animais, como caracterizado pelos resultados de pesquisas com caprinos $(5)$, bovinos $(6,7,8)$ e bubalinos (9).

Esta revisão tem como propósito realizar um levantamento dos estudos que avaliaram o papel da interação humano-animal na definição do bem-estar de ruminantes leiteiros, tratando de forma integrada a atitude dos manejadores em relação aos animais sob seus cuidados e a resposta dos animais aos manejos por eles realizados. Além disso, tem como objetivo avaliar

Andrioli M, Carvalhal M, Costa F, Costa MJRP. Efeitos da interação humano-animal no bem-estar de ruminantes leiteiros: Uma Revisão. Vet. e Zootec. 2020.; 27: 001-014. 
o papel modulador dos sistemas de produção adotados para as espécies de ruminantes produtoras de leite na definição da qualidade das interações entre humanos e animais.

\section{DESENVOLVIMENTO}

\section{Bem-estar animal e boas práticas de manejo}

O bem-estar animal é definido como o estado de um indivíduo em suas tentativas de lidar com os desafios de seu ambiente (10). Para Broom e Molento (11) este conceito referese a um estado mensurável que pode variar de muito bom a muito ruim; devendo-se ter em conta que para que um ambiente que ofereça condições para os animais apresentarem um bom estado de bem-estar, deve dispor de recursos que permitam o atendimento das necessidades de cada um dos indivíduos nele presente.

Um dado animal tem uma série de necessidades, que estão relacionadas com o seu funcionamento biológico e com a necessidades de obter acesso a recursos ou de evitar situações que o ameace (12), estando, portanto, diretamente relacionada com as condições de saúde, o estado fisiológico e de desenvolvimento dos animais, além da capacidade imunológica e a possibilidade de expressão de comportamentos naturais (13). Para Mellor e Reid (14), a avaliação do bem-estar animal deve levar em conta cinco domínios, quatro deles são definidos pelo estado físico do animal - envolvendo a nutrição, o ambiente, a saúde e o comportamento - e o quinto corresponde ao estado mental. Deve-se reconhecer que os efeitos sobre cada um desses domínios têm reflexos, diretos ou indiretos, nos demais, portanto, com efeitos recíprocos e aditivos.

Com o intuito de orientar o desenvolvimento das normas de bem-estar animal para os sistemas pecuários de produção a Organização Mundial de Saúde Animal (15) estabeleceu onze princípios gerais para orientar as ações que levam em conta o bem-estar dos animais de produção, como descrito a seguir: animais'.

1) 'A seleção genética deve sempre levar em consideração a saúde e o bem-estar dos

2) 'Os animais escolhidos para introdução em novos ambientes devem ser adequados ao clima local e capazes de se adaptar às doenças locais, parasitas e nutrição'.

3) 'O ambiente físico, incluindo o substrato (superfície de caminhada, superfície de descanso, etc.), deve ser adequado às espécies, de modo a minimizar o risco de lesões e a transmissão de doenças ou parasitas aos animais'.

4) 'O ambiente físico deve permitir repouso confortável, movimento seguro e confortável, incluindo movimentos normais, mudanças posturais e a oportunidade de realizar tipos de comportamento natural que os animais são motivados a realizar'.

5) ' $O$ agrupamento social de animais deve ser gerenciado para permitir um comportamento social positivo e minimizar lesões, angústias e medo crônico'.

6) 'Para animais alojados, a qualidade do ar, a temperatura e a umidade devem apoiar a boa saúde animal e não serem aversivas. Onde ocorrem condições extremas, os animais não devem ser impedidos de usar seus métodos naturais de termorregulação'.

7) 'Os animais devem ter acesso a alimentos e água suficientes, adequados à idade e às necessidades para manter a normalidade, saúde e produtividade e para evitar fome prolongada, sede, desnutrição ou desidratação'.

8) 'Doenças e parasitas devem ser prevenidos e controlados o máximo possível por meio de boas práticas de manejo. Os animais com sérios problemas de saúde devem ser isolados e tratados prontamente ou mortos humanamente se o tratamento não for viável ou a recuperação improvável'. 
9) 'Onde procedimentos dolorosos não puderem ser evitados, a dor resultante deve ser gerenciada na medida em que os métodos disponíveis permitam'.

10) ' $O$ manejo deve promover uma relação positiva entre humanos e animais e não deve causar lesões, pânico, medo duradouro ou estresse evitável'.

11) 'Proprietários e manejadores devem ter habilidade e conhecimento suficientes para garantir que os animais sejam tratados de acordo com esses princípios'.

Fica então evidente, em pelo menos um dos onze princípios estabelecidos, a importância da interação humano-animal no bem-estar dos animais de produção e, consequentemente, no estabelecimento de sistemas sustentáveis de produção pecuária; sendo reconhecido que as práticas de manejo possuem um papel central na definição do bem-estar dos animais de produção $(16,17)$.

Devido à relevância das interações entre humanos e animais, vários estudos têm sido realizados sobre o tema. Em um deles essas interações foram categorizadas como negativas (ou não amigáveis), positivas (ou amigáveis) ou neutras, quando não há interação (18). Sendo possível que essas interações ocorram envolvendo um dos cinco sentidos, tato, visão, olfato, paladar e audição $(19,20)$. Por conta disto, as interações podem ser classificadas conforme os sentidos envolvidos durante as interações entre os manejadores e os animais, sendo assim caracterizadas: 1) há apenas a presença visual do manejador; 2) há o deslocamento do manejador entre os animais, sem contato tátil, mas com possível utilização de interações vocais; 3) há contato físico não aversivo do manejador com os animais; 4) há oferta de alimento, considerada uma recompensa; e 5) há ocorrência de manejo aversivo (20). Além disso, qualidade do manejo vai depender tanto da natureza quanto da frequência das interações, por exemplo, quando é realizado um manejo aversivo, ou seja, que resulta em uma interação negativa, há alta probabilidade dos animais aprenderem a evitar situações semelhantes (e potencialmente perigosas) no futuro, apresentando reações de medo e dificultando o manejo, como caracterizado para bovinos por Rushen e colaboradores (21).

O medo resulta em estresse e, consequentemente, em efeitos negativos para o bem-estar animal, com potencial de prejudicar o crescimento e outras funções produtivas $(22,23)$, aspectos bem caracterizados para vacas leiteiras $(4,24)$. Por exemplo, há resultados de pesquisa (24) mostrando que a frequência e a natureza das interações táteis, visuais e auditivas do tratador em relação às vacas têm importante papel no comportamento desses animais durante a ordenha, bem como na produção e composição do leite, sendo evidenciado que as vacas que apresentavam reações de medo em relação aos humanos também apresentavam menor produção de leite, além de menores concentrações de gordura e proteína. Com relação à quantidade de leite residual nas vacas, há evidências de aumento de cerca de $70 \%$ quando na presença de um manejador aversivo durante a ordenha (21). Com base nesse resultado, é recomendável se evitar qualquer ação do manejador com potencial de provocar medo nas vacas.

Com o objetivo de avaliar a qualidade do manejo de vacas em lactação, Rosa e Paranhos da Costa (25) definiram quatro classes de interações entre os manejadores e as vacas leiteiras: 1. interação insignificante, quando os manejadores realizavam a ordenha sem interagir com a vaca; 2. interação desaconselhável, quando os manejadores apresentavam comportamentos negativos, como torcer a cauda, empurrar e bater nas vacas; 3 . interação instável, quando havia inconsistência na forma com que o manejador lidava com as vacas, apresentando interações positivas com as vacas em alguns momentos e negativas em outros; e 4. interação aconselhável, quando os manejadores apresentavam ações positivas durante a interação com as vacas. Como resultado, na interação considerada insignificante as vacas mantiveram-se apáticas, na interação desaconselhável os animais apresentaram reações típicas de medo e diminuíram a produção de leite em 1,0 kg de leite/animal/ordenha. Os resultados da interação instável foram parecidos com os da interação desaconselhável, diminuindo a 
produção de leite em 1,6 kg de leite/animal/ordenha, mesmo considerando que receberam ações positivas dos manejadores em algum momento. Já na interação aconselhável os animais não apresentaram queda de produção e melhoraram o nível de bem-estar, tendo como base indicadores comportamentais. Esses resultados mostram que as respostas de vacas leiteiras podem variar em relação ao tipo de interação com o humano, tendo em conta que quanto pior a interação, pior será a resposta das vacas, tanto comportamental quanto produtiva.

À interação humano-animal podemos incluir a possibilidade de os animais reconhecerem seres humanos específicos e essa capacidade influenciar a forma como os animais reagem a pessoas específicas de acordo com o manejo realizado por cada uma delas. Há evidências (18) de que bezerros bovinos podem discriminar diferentes pessoas com base em experiências anteriores, o que pode levar os animais a desenvolverem medo de pessoas específicas, que lhes causaram algum tipo de sofrimento. A discriminação de pessoas com base no manejo também foi descrita por outros autores (26) para vacas em lactação; os autores ainda relataram que a discriminação das pessoas por parte das vacas não é baseada exclusivamente na cor de suas roupas e no local onde o manejo aversivo ocorreu.

Com relação aos caprinos, é reconhecido que são animais que apresentam alto nível de atividade $(27,28)$, são exploradores, curiosos $(29,30)$ e têm grande capacidade de aprendizado, mantendo na memória as experiências vividas por longo prazo. Há também estudos com cabras $(31,32)$ que comprovaram que elas são capazes de discriminar com precisão os estímulos visuais aprendidos em uma tela, mesmo após várias semanas. Porém, é evidente que o foco das metodologias utilizadas nos estudos que buscam entender os efeitos da interação humano-animal sobre o comportamento dessa espécie é incipiente quando comparada às metodologias utilizadas nos estudos com bovinos. Por exemplo, existem alguns trabalhos que mostram que as cabras discriminam os seres humanos usando a visão (33) e a audição (34). No entanto, não encontramos estudos que descrevam com exatidão como e sob que circunstâncias as cabras discriminam ou percebem os sinais dos seres humanos $(29,35)$. Enfatizamos que esses estudos são importantes, pois permitirão determinar as práticas de manejo mais relevantes para espécie, evitando o estresse e prejuízos com relação ao bem-estar das cabras leiteiras.

Apesar da falta de informações mais detalhadas, é provável que a qualidade da interação humano-animal tenha grande influência no comportamento e na produtividade das cabras leiteiras. Por exemplo, em um estudo conduzido por Kaminski e colaboradores (36), os autores tinham como objetivo identificar diferenças no comportamento de caprinos submetidos a um alto grau de interação humana (o manejador entrava na baia duas vezes por dia, andando calmamente por 20 minutos entre as cabras) e a um baixo grau de interação (o manejador entrava na baia todos os dias somente para limpar os bebedouros). Todos os animais foram avaliados por meio do teste de velocidade de saída (velocidade média que as cabras saíam da instalação que estavam contidas e se afastavam das pessoas), pela expressão de comportamentos agressivos e pela avaliação qualitativa do comportamento, que busca avaliar a linguagem corporal dos animais como um indicador de bem-estar animal, como descrito por Ceballos e Sant'Anna (37). Com esses testes os autores constataram a habituação das cabras ao manejador andando entre as baias, promoveu efeitos positivos no bem-estar e no desempenho produtivo desses animais, que expressaram menos comportamentos agonísticos, menor velocidade de saída e maior massa corporal (36).

De maneira similar, foi realizado um estudo com o objetivo de propor um conjunto de ações simples e práticas para habituar pequenos ruminantes das raças Pelibuey (ovinos) e Criollo (caprinos) à presença contínua de observadores humanos. Os animais foram classificados em dois grupos: (1) fêmeas ovinas que apresentaram comportamento de esquiva ao primeiro contato com o observador e (2) fêmeas caprinas que apresentaram o comportamento de seguir o observador ao primeiro contato. $\mathrm{O}$ primeiro teste de habituação 
foi aplicado para os animais com comportamento de esquiva e começou com o caminhar do observador em direção aos animais e parou quando $10 \%$ dos animais começaram a evitá-lo, nesse momento foi medida a distância do animal mais próximo do observador. O segundo teste de habituação foi utilizado nos animais que seguiram o observador. Era realizado quando um observador entrava na baia e esperava por um minuto até que os animais se aproximassem dentro de um raio de um metro. Então, esperava por dois minutos, e o observador mudava a posição para mais distante dos animais até que o número de seguidores fosse reduzido. Ambos os testes foram feitos nos animais alojados em piquetes e em baias. A habituação foi considerada bem-sucedida quando todos os animais do grupo podiam ser observados de uma distância de um metro, sem evitar e sem seguir o observador. Como resultado, foi observada uma diminuição da distância de fuga (distância mínima que o animal permite aproximação) e redução dos animais seguidores. Nos ovinos, o tempo de sucesso no processo de habituação foi de doze dias na baia e dez dias no piquete, enquanto nos caprinos foi de treze dias na baia e dez dias no piquete. Ao final do estudo foi elaborado um protocolo prático para pequenos ruminantes se habituarem à observação direta de seres humanos, o que deve auxiliar os estudos na área de etologia para essas espécies animais (38).

Nas búfalas, por sua vez, há evidências de que pequenas mudanças de rotina na ordenha influenciam a liberação de ocitocina e a produção de leite devido à sensibilidade desses animais na ordenha (39). Devido a características fisiológicas e anatômicas, as búfalas geralmente são consideradas mais difíceis de serem ordenhadas quando comparadas às vacas bovinas $(40,41)$. Por isso, muitos produtores, quando não realizam a ordenha com bezerro ao pé, aplicam ocitocina como estímulo para a ejeção do leite durante a ordenha. De forma complementar, há resultados mostrando que atitudes positivas dos manejadores durante a ordenha estão inversamente correlacionadas com a expressão de passos e coices, ou seja, quanto mais interações positivas com as búfalas, menor o número de coices expressados durante o manejo de ordenha (42). Esses autores descrevem também haver uma correlação positiva e moderada entre a aplicação de ocitocina e a expressão de passos e coices. Resultado que reforça a expectativa de que a aplicação de ocitocina um possível indicador de bem-estar referente a uma interação humano-animal inadequada (43). No Brasil, esses animais são considerados rústicos o que geralmente implica em menor atenção para o estado de bem-estar desses animais, pois os produtores acreditam que as búfalas são muito resistentes e que, por conta disso, não sofrem dor induzida pelo manejo (40). Uma das maneiras de aumentar a produtividade em búfalas, assim como, de todas as outras espécies criadas para produção de leite, é fazer com que a rotina do contato diário do animal com os humanos seja positiva.

Em estudos com a habituação de novilhas à rotina da ordenha foram encontrados resultados positivos em bovinos e bubalinos. Normalmente esses estudos envolvem a habituação dos animais à rotina de manejo de ordenha, caracterizada pela interação humanoanimal positiva e/ou neutra. Por exemplo, em um estudo em que foram avaliadas 72 novilhas bovinas das raças Pardo-Suíça, Holandês e Simental foram aplicados dois tratamentos, definidos pelas novilhas de passarem por um treinamento (que consistia em passar pela sala de ordenha e ficar expostas aos ruídos de ventiladores e ordenhadeira e ao contato tátil no úbere) e as que não passaram, sendo expostas à rotina de ordenha pela primeira vez após o parto. As novilhas treinadas se habituaram à sala de ordenha e apresentaram menores frequências de passos e coices (44). De maneira semelhante, Polikarpus (9) avaliaram 16 búfalas, sendo que oito delas foram treinadas dez dias antes da data provável de parição. As novilhas que receberam treinamento foram deixadas por dez minutos na sala de ordenha uma vez ao dia e, nessa oportunidade, o úbere era lavado, com toques suaves dos manejadores. Como resultado, as búfalas treinadas, quando ordenhadas após o parto, pateavam e coiceavam menos que aquelas não treinadas, mostrando que quando os animais são habituados a um manejo positivo há redução de comportamentos relacionados à inquietação dos mesmos.

Andrioli M, Carvalhal M, Costa F, Costa MJRP. Efeitos da interação humano-animal no bem-estar de ruminantes leiteiros: Uma Revisão. Vet. e Zootec. 2020.; 27: 001-014. 
As interações entre humanos e animais podem ter efeitos significativos na produção animal, principalmente quando se trata de animais com contanto diário, como nas cadeias produtivas da pecuária de leite. Os estudos comprovam que atitudes positivas e negativas por parte dos manejadores, influenciam positivamente e negativamente o comportamento e a produtividade dos animais. Do mesmo modo, a habituação e o treinamento dos animais podem ser usados como uma estratégia para promover a interação positiva entre humanos e animais, com efeitos positivos no bem-estar animal.

\section{Viabilização da interação humano-animal positiva}

A interação positiva reflete de forma benéfica no comportamento, na fisiologia e consequentemente, no bem-estar dos animais de produção e dos manejadores; com possibilidades de reduzir o estresse, a incidência de doenças e melhorar os índices produtivos $(17,45)$. Existem diversos resultados satisfatórios quanto às melhorias nas respostas comportamentais e produtivas de animais, como caracterizado em resultados de pesquisa com bezerras leiteiras, que mostraram efeitos positivos a longo prazo no comportamento de bezerras submetidas a boas práticas de manejo. Para o estudo, os autores avaliaram do nascimento até a desmana, o comportamento, a saúde e o desempenho de 98 bezerras mestiças das raças Holandês e Gir divididas em três tratamentos: boas práticas de manejo com escovação, boas práticas de manejo e o grupo controle. As boas práticas de manejo consistiram em desinfecção do umbigo dentro de 3 horas após o nascimento, alimentação por colostro com qualidade assegurada, fornecimento do leite em baldes com bico e acompanhamento diário visando à identificação de qualquer sinal clínico de doenças. Foram encontrados efeitos positivos ao longo prazo no comportamento das novilhas leiteiras mestiças (46). Dessa forma, os achados indicaram que animais manejados sob boas práticas de manejo podem levar os efeitos positivos a longo prazo, de modo a facilitar o manejo diário nas fazendas, reduzindo o tempo de trabalho. Resultados semelhantes foram obtidos com vacas, demonstrando que as interações positivas entre seres humanos e vacas podem diminuir o estresse causado por procedimentos veterinários de rotina como palpação retal e inseminação artificial (47).

A interação humano-bovino, das mais variadas formas (tátil, visual, olfativa, gustativa e auditiva) parecem ser relevantes na qualidade de vida dos animais, demonstrando um efeito recíproco no humano e no animal (27); como também demonstrado no estudo que avaliou os efeitos das atitudes dos manejadores (falar e tocar) no comportamento e na produção de vacas em 30 fazendas. Os autores observaram que o comportamento dos manejadores estava fortemente correlacionado com o comportamento das vacas e moderadamente correlacionado com a produção de leite. As interações positivas, como acariciar e falar calmamente, fizeram com que as vacas não evitassem os manejadores na ordenha; enquanto gritos, bater com varas e com as mãos fizeram com que as vacas evitassem os manejadores, dificultando o trabalho e diminuindo a produção de leite (47).

Os primeiros estudos sobre os efeitos da interação humano-animal positiva, caracterizada pela estimulação tátil, demonstraram que ratos estimulados na infância abriram os olhos, apresentaram capacidade locomotora e foram desmamados mais cedo quando comparados aos animais que não receberam essa estimulação (48). Embora esse estudo tenha sido realizado com ratos, alguns autores sugerem que resultados semelhantes poderiam ser encontrados com neonatos de outras espécies, inclusive com não mamíferos, como no caso das aves (49). Desde então, os estudos nessa área avançaram comprovando os benefícios da interação humano-animal positiva. Há evidências que essas ações devem iniciar bem cedo na vida dos animais, durante a fase sensível para aprendizado. Por exemplo, Magalhães Silva (50) descreveu que a adoção da estimulação tátil em bezerros cruzados (Holandês e Gir), logo 
nos primeiros dias após o nascimento, foi eficiente para a formação de laços com humanos, com efeitos positivos na saúde e no desenvolvimento dos bezerros e, como mencionado anteriormente, podem permanecer por longo prazo (46). Além disso, é comprovado que a estimulação tátil promove o relaxamento nos animais de diferentes espécies, por meio da diminuição da frequência cardíaca e expressão de comportamentos como poucos movimentos corporais, olhos fechados e cabeça baixa em cavalos, ratos e bovinos (51-53).

Em estudo com caprinos, Andrioli (54) avaliou os efeitos da estimulação tátil na saúde e no desempenho de 36 cabritos da raça Saanen do $5^{\circ}$ ao $40^{\circ}$ dia de idade. Nesse estudo, foi observada a diminuição da ocorrência de corrimento nasal, durante os primeiros 30 dias de vida, no grupo de animais estimulados quando comparados aos animais não estimulados, além de diferença significativa com relação ao ganho de peso aos 15 dias de idade, os cabritos estimulados ganharam 280 gramas a mais que aqueles não estimulados. Com esses resultados podemos perceber que a estimulação tátil interfere positivamente no período sensível da vida do animal (do nascimento ao desmame), melhorando o grau de bem-estar dos animais por meio da promoção da saúde dos mesmos e, consequentemente, diminuindo os gastos com medicamentos veterinários, garantindo maior lucratividade ao produtor.

Ainda com relação aos pequenos ruminantes, Jackson e Hacket (45) testaram a estimulação tátil realizada em cabras leiteiras por 10 minutos durante 24 dias. Os autores observaram que as cabras que foram estimuladas apresentaram menor latência de aproximação e menor o tempo de habituação aos humanos quando comparadas com as cabras do grupo controle. Desse modo, podemos afirmar que simples mudanças na rotina de manejo das propriedades podem auxiliar em trabalhos diários como a ordenha.

Outra ferramenta que vem sendo utilizada para melhorar a interação humano-animal e o bem-estar dos animais de produção é a intervenção multissensorial. Em um estudo realizado com bezerras cruzadas Holandês x Gir (55), os autores descreveram que essa intervenção no período de desmame (por meio de afagos na cabeça, pescoço, linha dorsal, flancos, nos membros, na inserção da cauda e no úbere, além dos animais serem chamados pelo próprio nome em tom de voz tranquilo por uma pessoa que manteve o contato visual) é possível de ser feita e tem importante papel para melhorar o bem-estar dos animais. Com bases nesses resultados recomenda-se realizar mais estudos que visem avaliar os benefícios dessa prática para as diversas espécies leiteiras.

De forma geral, os resultados da boa interação humano-animal indicam haver efeitos positivos na produtividade e no bem-estar dos animais, como caracterizado pela redução da frequência cardíaca (56), aumento da expressão de docilidade (57), facilitação da formação do vínculo entre humanos e animais $(58,59)$ e facilitação do manejo (16). Estabelecer protocolos práticos para executar a relação humano-animal positiva no dia-a-dia das fazendas é um desafio para as cadeias produtivas da pecuária leiteira. No entanto, pequenas mudanças nas rotinas do manejo como, por exemplo, a adoção de boas práticas e estimulação multissensorial podem melhorar o bem-estar dos animais e facilitar o trabalho dos manejadores.

\section{CONSIDERAÇÕES FINAIS}

No Brasil, as cadeias produtivas das principais espécies produtoras de leite (bovinos, caprinos, ovinos e bubalinos) contribuem substancialmente na balança comercial do país. Com base nos trabalhos expostos nessa revisão há maneiras de melhorar o bem-estar desses animais independentemente do tipo de sistema de criação, sendo a promoção de interações positivas entre humanos e animais uma das formas de se conseguir isto.

Essa estreita relação entre humanos e animais pode ser desenvolvida por meio do uso de interações positivas envolvendo um ou vários dos cinco sentidos, tato, visão, olfato, 
paladar e audição. Algumas vezes, a simples presença do manejador andando calmamente entre os animais já traz benefícios. Quando isso é associado ao uso de ações positivas durante a interação como falar calmamente, tocar e acariciar os animais, oferecer alimento como recompensa ou mesmo a utilização de boas práticas de manejo associadas à estimulação tátil com escovação, os resultados positivos podem ser potencializados. O importante é desenvolver uma rotina de contato positivo diário entre humanos e animais. Outras estratégias, como a habituação à rotina da ordenha e o treinamento das fêmeas jovens, por exemplo, também devem ser estimuladas a fim de promover o bem-estar dos animais e das pessoas envolvidas no manejo, bem como propiciar ganhos na produção e na qualidade do leite, facilitando ainda a detecção de problemas de saúde. Cabe ressaltar a importância de treinar as pessoas responsáveis pelo manejo, tornando-as capazes de compreender o comportamento dos animais e identificar aspectos que possam prejudicar o bem-estar dos mesmos.

Como evidenciado nesta revisão, ainda são escassos os estudos relacionados ao bemestar de ovinos, caprinos e bubalinos, o que enfatiza a importância de aumentar e aprofundar os estudos sobre o bem-estar dessas espécies, com o propósito de melhorar a qualidade de vida desses animais, bem como seus índices produtivos. Dessa forma incentivamos mais estudos que tenham como objetivo melhorar a relação humano-animal, adotando as boas práticas de manejo na produção das espécies leiteiras. Esperamos então, termos levantado algumas questões ainda não respondidas que poderão dar abertura a pesquisas futuras, dando continuidade à construção do conhecimento nesta área.

\section{REFERÊNCIAS}

1. Broom DM. Animal welfare in the European Union [Internet]. Brussels: DirectorateGeneral for Internal Policies (Policy Department, Citizen's Rights and Constitutional Affairs); 2017 [cited 2017 Jan 19]. Available from: https://www.europarl.europa.eu/RegData/etudes/STUD/2017/583114/IPOL_STU(2017)58 3114_EN.pdf

2. Broom DM. Animal welfare: an aspect of care, sustainability, and food quality required by the public. J Vet Med Educ. 2010;37(1):83-8. doi: https://doi.org/10.3138/jvme.37.1.83.

3. Broom DM. Animal welfare complementing or conflicting with other sustainability issues. Appl Anim Behav Sci. 2019;219:104829. doi: https://doi.org/10.1016/j.applanim.2019.06.010.

4. Hemsworth PH, Coleman GJ, Barnett JL. The effects of cognitive behavioral intervention on the attitude and behavior of stockpersons and the behavior and productivity of commercial dairy cows. J Anim Sci. 2002;80(1):68-78. doi: https://doi.org/10.2527/2002.80168x.

5. Baxter EM, Mulligan J, Hall SA, Donbavand JE, Palme R, Aldujaili E, et al. Positive and negative gestational handling influences placental traits and mother-offspring behavior in dairy goats. Physiol Behav. 2016;157:129-38. doi: https://doi.org/10.1016/j.physbeh.2016.02.001.

6. Jago JG, Krohn CC, Matthews LR. The influence of feeding and handling on the development of the human-animal interactions in young cattle. Anim Behav Sci. 1999;62(2-3):137-51. doi: https://doi.org/10.1016/S0168-1591(98)00219-6. 
7. Lensink BJ, Boivin X, Pradel P, Le Neindre P, Veisser I. Reducing veal calves' reactivity to people by providing additional human contact. J Anim Sci. 2000;78(5):1213-8. doi: https://doi.org/10.2527/2000.7851213x.

8. Lensink BJ, Fernadez X, Boivin X, Pradel P, Le Neindre P, Veisser I. The impact of gentle contacts on ease of handling, welfare, and growth of calves and on quality of veal meat. $\mathrm{J}$ Anim Sci. 2000;78(5):1219-26. doi: https://doi.org/10.2527/2000.7851219x.

9. Polikarpus A, Napolitano F, Grasso F, Di Palo R, Zicarelli F, Arney D, et al. Effect of prepartum habituation to milking routine on behaviour and lactation performance of buffalo heifers. Appl Anim Behav Sci. 2014;161:1-6. doi: https://doi.org/10.1016/j.applanim.2014.10.003.

10. Broom DM. Indicators of poor welfare. Br Vet J [Internet]. 1986 [cited 2017 Jan 19];142(6):524-6. Available from: https://endcap.eu/wp-content/uploads/2015/06/Broom1986-Indicators-of-poor-animal-welfare.pdf

11. Broom DM, Molento CFM. Bem-estar animal: conceito e questões relacionadas revisão. Arch Vet Sci [Internet]. 2004 [cited 2017 Jan 19];9(2):1-11. Available from: https://revistas.ufpr.br/veterinary/article/viewFile/4057/3287

12. Broom D. Animal welfare: concepts, study methods and indicators. Rev Colomb Cienc Pecu [Internet]. 2011 [cited 2017 Jan 19];24(3):306-321. Available from http://www.scielo.org.co/pdf/rccp/v24n3/v24n3a10.pdf

13. Blokhuis HJ, Veissier I, Miele M, Jones B. The Welfare Quality® project and beyond: safeguarding farm animal well-being. Acta Agric Scand A Anim Sci. 2010;60(3):129-40. doi: https://doi.org/10.1080/09064702.2010.523480.

14. Mellor DJ, Reid CSW. Concepts of animal well-being and predicting the impact of procedures on experimental animals. WBI Stud Repository [Internet]. 1994 [cited 2017 Jan 19]:3-18. Available from: https://animalstudiesrepository.org/exprawel/7/

15. World Organisation for Animal Health - OIE. Terrestrial animal health code. Paris: OIE; 2019 [cited 2017 Jan 19]. Introduction to the recommendations for animal welfare [Internet]; sec. 7 (Animal Welfare), chap. 7.1, p. 1-4. Available from: https://www.oie.int/fileadmin/Home/eng/Health_standards/tahc/current/chapitre_aw_intr oduction.pdf

16. Boivin X, Le Neidre P, Chupin JM. Establishment of cattle-human relationships. Anim Behav Sci. 1992;32(4):325-35. doi: https://doi.org/10.1016/S0168-1591(05)80025-5.

17. Hemsworth PH. Human-animal interactions in livestock production. Appl Anim Behav Sci. 2002;81(3):185-98. doi: https://doi.org/10.1016/S0168-1591(02)00280-0.

18. de Passillé AM, Rusehn J, Ladewig J, Petherick C. Dairy calves discrimination of people based on previous handling. J Anim Sci. 1996;74(5):969-74. doi: https://doi.org/10.2527/1996.745969x.

Andrioli M, Carvalhal M, Costa F, Costa MJRP. Efeitos da interação humano-animal no bem-estar de ruminantes leiteiros: Uma Revisão. Vet. e Zootec. 2020.; 27: 001-014. 
19. Honorato LA, Hotzel MJ, de Miranda Gomes CC, Silveira IDB, Machado Filho LCP. Particularities of the human-animal interactions relevant to the welfare and productivity of dairy cows. Cienc Rural. 2012;42(2):332-40.

20. Waiblinger S, Bovin X, Perdersen V, Tosi MV, Janczak AM, Visser EK, et al. Assessing the human-animal relationship in farmed species: a critical review. Appl Anim Behav Sci. 2006;101(3-4):185-242. doi: https://doi.org/10.1016/j.applanim.2006.02.001.

21. Rushen J, Taylor AA, de Passilé AA. Domestic animals' fear of humans and its effect on their welfare. Appl Anim Behav Sci. 1999;65(3):285-303. doi: https://doi.org/10.1016/S0168-1591(99)00089-1.

22. Hemsworth PH, Barnett JL, Coleman GJ. The human-animal relationship in agriculture and its consequences for the animal. Anim Welf. 1993;2(1):33-51.

23. Grandin T. Solving livestock handling problems. Vet Med [Internet]. 1994 [cited 2017 Jan 19];89(10):989-98. Available from: https://www.researchgate.net/profile/Temple_Grandin/publication/266589526_Solving_li vestock_handling_problems_Solving_livestock_handling_problems/links/5559eebc08aea aff3bfab46f.pdf

24. Breuer K, Hemsworth PH, Barnett JL, Matthews LR, Coleman GJ. Behavioural response to humans and the productivity of commercial dairy cows. Appl Anim Behav Sci. 2000;66(4):273-88. doi: https://doi.org/10.1016/S0168-1591(99)00097-0.

25. Rosa MS, Paranhos da Costa MJR. O reflexo da interação amigável entre humanos e bovinos no bem-estar na fazenda. In: Anais do 6o Congresso Brasileiro de Raças Zebuínas; 2005; Uberaba. Uberaba: ABCZ; 2005. p.71-9.

26. Munksgaard L, de Passillé AM, Rushen J, Thodberg K, Jensen MB. Discrimination of people by dairy cows based on handling. J Dairy Sci. 1997;80(6):1106-12. doi: https://doi.org/10.3168/jds.S0022-0302(97)76036-3.

27. Kilgour R, Dalton C. Livestock behavior: a practical guide. London: Granada Publishing; 1984.

28. Silva CMD, Furtado DA, Medeiros AND, Saraiva EP, Guimarães MCDC, Tota LDCA, et al. Ethogram of three genetic groups of goats confined using monitoring video images. Rev Etol. 2013;12(1):1-11.

29. Nawroth C. Invited review: Socio-cognitive capacities of goats and their impact on human-animal interactions. Small Rumin Res. 2017;150:70-5. doi: https://doi.org/10.1016/j.smallrumres.2017.03.005.

30. Langbein J, Siebert K, Nürnberg G. On the use of an automated learning device by group-housed dwarf goats: do goats seek cognitive challenges? Appl Anim Behav Sci. 2009;120(3-4):150-8. doi: https://doi.org/10.1016/j.applanim.2009.07.006.

Andrioli M, Carvalhal M, Costa F, Costa MJRP. Efeitos da interação humano-animal no bem-estar de ruminantes leiteiros: Uma Revisão. Vet. e Zootec. 2020.; 27: 001-014. 
31. Langbein J, Nürnberg G, Manteuffel G. Visual discrimination learning in dwarf goats and associated changes in heart rate and heart rate variability. Physiol Behav. 2004;82(4):601-9. doi: https://doi.org/10.1016/j.physbeh.2004.05.007.

32. Langbein J, Siebert K, Nuernberg G. Concurrent recall of serially learned visual discrimination problems in dwarf goats (Capra hircus). Behav Processes. 2008;79(3):15664. doi: https://doi.org/10.1016/j.beproc.2008.07.004

33. Keil NM, Imfeld-Mueller S, Aschwanden J, Wechsler B. Are head cues necessary for goats (Capra hircus) in recognising group members? Anim Cogn [Internet]. 2012 [cited 2017 Jan 19];15(5):913-21. Available from: https://link.springer.com/article/10.1007/s10071-012-0518-6

34. Briefer EF, Padilla de la Torre M, McElligott AG. Mother goats do not forget their kids' calls. Proc Biol Sci. 2012;279(1743):3749-55. doi: https://doi.org/10.1098/rspb.2012.0986.

35. Kaminski J, Riedel J, Call J, Tomasello M. Domestic goats, Capra hircus, follow gaze direction and use social cues in an object choice task. Anim Behav. 2005;69(1):11-18. doi: https://doi.org/10.1016/j.anbehav.2004.05.008.

36. Miller DW, Fleming PA, Barnes AL, Wickham SL, Collins T, Stockman CA. Behavioural assessment of the habituation of feral rangeland goats to an intensive farming system. Appl Anim Behav Sci. 2018;199:1-8. doi: https://doi.org/10.1016/j.applanim.2017.11.001.

37. Ceballos MC, Sant'Anna AC. Evolução da ciência do bem-estar animal: aspectos conceituais e metodológicos. Rev Acad Cienc Anim. 2018;16:1-24. doi: 10.7213/19814178.2018.161103.

38. Gonzaléz-Pech PG, Marín-Tun CG, Valladares-González DA, Ventura-Cordero J, OrtízOcampo GI, Cámara-Sarmiento R, et al. A protocolo $\mathrm{f}$ human animal interaction to habituate young sheep and goats for behavioural studies. Behav Processes. 2018;157:6327. doi: https://doi.org/10.1016/j.beproc.2018.04.007.

39. Thomas CS, Bruckmaier RM, Östensson K, Svennersten-Sjaunja K. Effect of different milking routines on milking related release of the hormones oxytocin, prolactin and cortisol, and on milk yield and milking performance in Murrah buffaloes. J Dairy Res. 2005;72(1):10-8. doi: https://doi.org/10.1017/S0022029904000457.

40. Barbosa Neto JD, Bastianetto E. Diferenças fisiológicas entre bubalinos e bovinos: interferência na produção. Cienc Anim Bras [Internet]. 2009 [cited 2020 Jul 19];10. Available from: https://www.revistas.ufg.br/vet/article/view/7664

41. Carvalhal MVL, Costa FO. Produção e bem-estar de búfalas (Bubalus bubalis) leiteiras: uma revisão. Rev Acad Cienc Anim [Internet]. 2018 [cited 2020 Jul 19];16:1-10. Available from:

https://www.bufalo.com.br/home/wpcontent/uploads/2016/01/dramonique.pdf

42. Saltalamacchia F, Tripaldi C, Castellano A, Napolitano F, Musto M, De Rosa G. Human and animal behaviour in dairy buffalo at milking. Anim Welf [Internet]. 2007 [cited 2020 
Jul 19];16(2):139.

Available

from:

https://www.researchgate.net/profile/Giuseppe_Rosa/publication/233583710_Human_an d_animal_behaviour_in_dairy_buffalo_at_milking/links/09e4150c853a30ad44000000/H uman-and-animal-behaviour-in-dairy-buffalo-at-milking.pdf

43. De Rosa G, Napolitano F, Grasso F, Pacelli C, Bordi A. On the development of a monitoring scheme of buffalo welfare at farm level. Ital J Anim Sci. 2005;4(2):115-25. doi: https://doi.org/10.4081/ijas.2005.115.

44. Kutzer T, Steilen M, Gygax L, Wechsler B. Habituation of dairy heifers to milking routine-Effects on human avoidance distance, behavior, and cardiac activity during milking. J Dairy Sci. 2015;98(8):5241-51. doi: https://doi.org/10.3168/jds.2014-8773.

45. Jackson KMA, Hackett D. A note: the effects of human handling on heart girth, behaviour and milk quality in dairy goats. Appl Anim Behav Sci. 2007;108(3-4):332-6. doi: https://doi.org/10.1016/j.applanim.2007.01.011.

46. Silva LP, Sant'anna AC, Silva LCM, Paranhos da Costa MJR. Long-term effects of good handling practices during the pre-weaning period of crossbred dairy heifer calves. Trop Anim Health Prod [Internet]. 2017 [cited 2020 Jul 19];49(1):153-62. Available from: https://link.springer.com/article/10.1007/s11250-016-1174-7

47. Waiblinger S, Menke C, Coleman G. The relationship between attitudes, personal characteristics, behavior of stockpeople, subsequent behavior, and production of dairy cows. Appl Anim Behav Sci. 2002;79(3):195-219. doi: https://doi.org/10.1016/S01681591(02)00155-7.

48. Levine S, Haltmeyer GC, Karas GG, Denenberg VH. Physiological and behavioral effects of infantile stimulation. Physiol Behav. 1967;2(1):55-9. doi: https://doi.org/10.1016/0031-9384(67)90011-X.

49. Smith FV, Nott KH. The 'critical period' in relation to the strength of the stimulus. Z Tierpsychol. 1970;27(1):108-115. doi: https://doi.org/10.1111/j.14390310.1970.tb01865.x.

50. Magalhães Silva LC. Avaliação dos benefícios da adoção de boas práticas de manejo no bem-estar de bezerros leiteiros [tese]. Jaboticabal: Faculdade de Ciências Agrárias e Veterinárias, Universidade Estadual Paulista; 2015.

51. Kurowasa M, Lundeberg T, Agren G, Lund I, Uvnas-Moberg K. Massage-like stroking of the abdomen lowers blood pressure in anesthetized rats: Influence of oxytocin. J Auton Nerv Syst. 1995;56(1-2):26-30. doi: https://doi.org/10.1016/0165-1838(95)00056-7.

52. Lynch JJ, Frederick Fregin G, Mackie JB, Monroe Jr RR. Heart rate changes in the horse to human contact. Psychophysiology. 1974;11(4):472-8. doi: https://doi.org/10.1111/j.1469-8986.1974.tb00575.x.

53. Schmied C, Waiblinger S, Scharl T, Leisch F, Boivin X. Stroking of different body regions by a human: effects on behaviour and heart rate of dairy cows. Appl Anim Behav Sci. 2008;109(1):25-38. doi: https://doi.org/10.1016/j.applanim.2007.01.013.

Andrioli M, Carvalhal M, Costa F, Costa MJRP. Efeitos da interação humano-animal no bem-estar de ruminantes leiteiros: Uma Revisão. Vet. e Zootec. 2020.; 27: 001-014. 
54. Andrioli M. Efeito da estimulação tátil no bem-estar de cabritos leiteiros [trabalho de conclusão de curso]. Jaboticabal: Faculdade de Ciências Agrárias e Veterinárias, Universidade Estadual Paulista; 2018.

55. Valente PP, Almeida DHS, Paranhos da Costa MJR. Técnica da estimulação multissensorial em bezerras Girolando. In: Anais do Encontro Anual de Etologia; 2016; Jaboticabal. Jaboticabal: Faculdade de Ciências Agrárias e Veterinárias; 2016. p.105.

56. Waiblinger S, Menke C, Korff J, Bucher A. Previous handling and gentle interactions affect behaviour and heart rate of dairy cows during a veterinary procedure. Appl Anim Behav Sci. 2004;85(1-2):31-42. doi: https://doi.org/10.1016/j.applanim.2003.07.002.

57. Arave CW, Mickelsen CH, Walters JL. Effect of early rearing experience on subsequent behavior and production of Holstein heifers. J Dairy Sci. 1985;68(4):923-9. doi: https://doi.org/10.3168/jds.S0022-0302(85)80910-3.

58. Hemsworth PH, Barnett JL, Hansen C. The influence of inconsistent handling by humans on the behaviour, growth and corticosteroids of young pigs. Appl Anim Behav Sci. 1987;17(3-4):245-52. doi: https://doi.org/10.1016/0168-1591(87)90149-3.

59. Hemsworth PH, Barnett JL. Relationships between fear of humans, productivity and cage position of laying hens. $\mathrm{Br}$ Poult Sci. 1989;30(3):505-18. doi: https://doi.org/10.1080/00071668908417175.

Recebido em: 30/07/2020

Aceito em: 07/12/2020 